
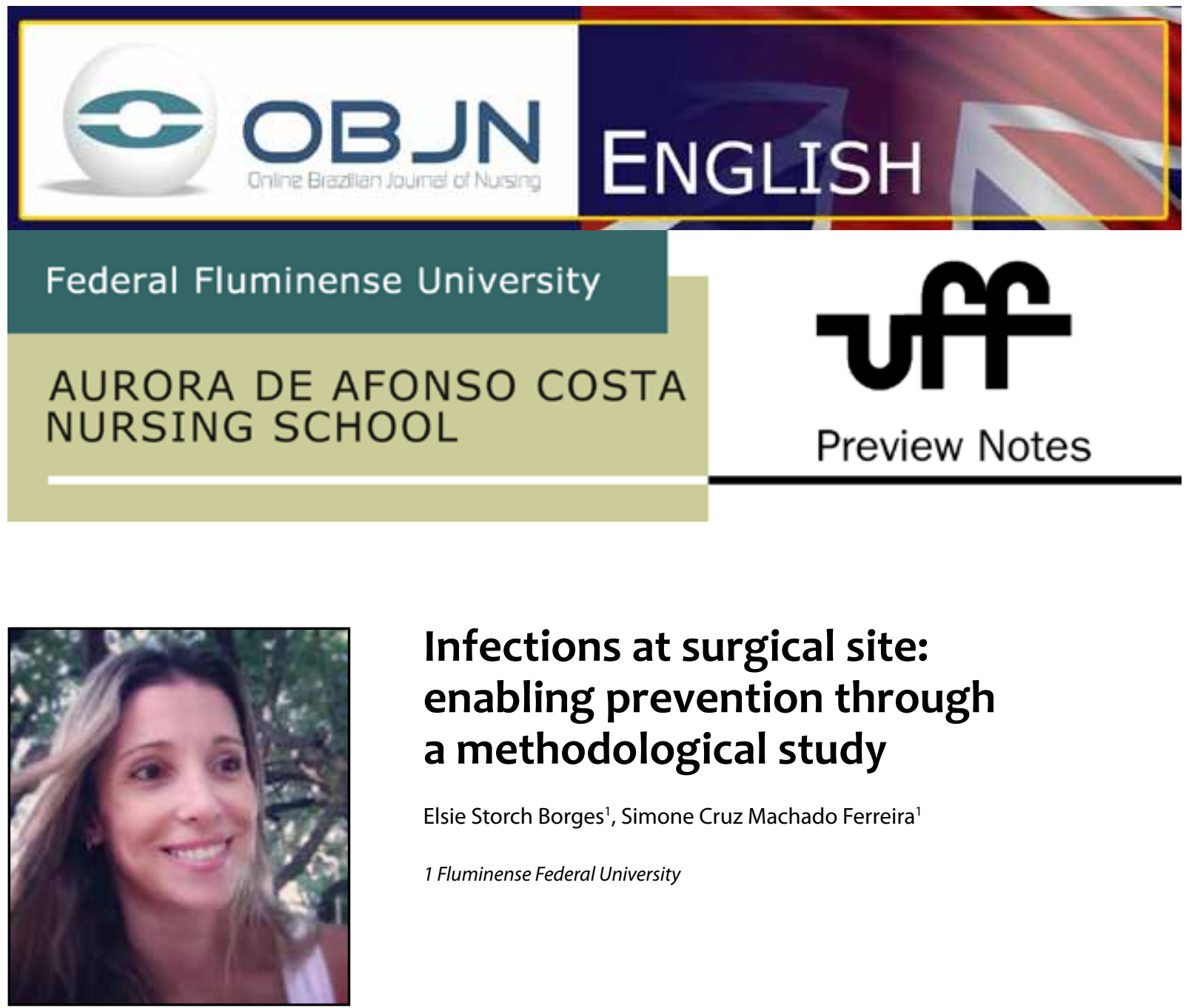

\title{
Infections at surgical site: enabling prevention through a methodological study
}

\author{
Elsie Storch Borges' ${ }^{1}$ Simone Cruz Machado Ferreira' \\ 1 Fluminense Federal University
}

\section{ABTRACT}

This is a dissertation project, part of the Professional Master's in Assisting Nursing. Aim: to identify, based on the literature, the most relevant actions to control Infection at Surgical Sites (ISS); to elaborate an instrument to evaluate the most relevant actions to prevent and control ISS as used by the Control Commission of Hospital Infection (CCHI); and to validate the instrument, applying it at the researched scenario. Method: this is a methodological study, which adopts a qualitative approach. The aim is to acquire, analyze and organize the information gathered regarding ISS in an integrative review of the scientific literature found on Lilacs and Mediline database, over a time period of 10 years. As a result of this research, an instrument will be produced and used daily at the CCHI. It will be tested by specialist professionals at the scene of application. The validation will be given by consensus of a group chosen for this purpose.

Descriptors: Surgical Procedures, Operative; Surgical Wound Infection; Risk Factors; Patient Safety; Checklist. 


\section{PROBLEM SITUATION AND ITS SIGNIFICANCE}

Today, patient safety is one of the most critical situations for the state of health of Brazil. Practice based on evidence has been widely used due to a growing need to reduce avoidable complications, and to prevent occasional errors, permitting the reduction of undesired negative outcomes. In this context, infections related to health assistance (IRHA) are considered to be avoidable adverse events ${ }^{(1)}$. Among the IRHA, the infections at surgical sites (ISS) have been increasingly emphasized, both in Brazil and around the world, because of its influence, as well as the financial impact due to the negative outcomes on the part of patients. ISS occur where surgery has been carried out, located in the superficial or deep layers of the incision, organs or the part of the body that has been manipulated or traumatized, such as the peritoneal, pleural, mediastinal or articular spaces. According to information collected worldwide, ISS contribute to around $15 \%$ of all infections related to health care assistance, and $37 \%$ of the ones acquired in hospitals by surgical patients ${ }^{(2)}$. The Control Commissions of Hospital Infection (CCHI) have to systematize and perform all sorts of actions that have a direct impact in reducing IRHA - which is not always an easy task, due to its complexity and specificity of each scenario, especially when it is related to the practices of nurses ${ }^{(3)}$. The goal of this study is the development of an instrument to evaluate the degree of conformity of actions related to the control and prevention of ISS. This instrument will be produced from the identification of the actions which have a particularly great impact on the control of such illnesses, based on today's literature, according to the concepts of patient safety, as the described at the program Safe Surgery. There is an intention that this instrument will be used by professionals who work in $\mathrm{CCHI}$, who will be able to use it systematically in an attempt to improve and make each item adequate, as well as to correlate the local ISS levels with the other points described in the literature. The validation of the instrument will also be done as part of the process of the development of this research.

\section{GUIDING QUESTIONS}

Which are the most relevant concepts and which have the greatest impact with regard to the prevention and control of ISS? How can we use these concepts in order to identify the most relevant actions which have the greatest impact in preventing and controlling ISS as part of the daily practices at the $\mathrm{CCHI}$ ? Is the proposed instrument sufficiently effective when applied in practice?

\section{AIMS}

I) To identify, based on today's literature, the most relevant actions to control ISS;

II) To elaborate an instrument for use by the Control Commission of Hospital Infection to evaluate the most relevant actions to prevent and control ISS;

III) To validate the instrument created, using it at the scenario under consideration.

\section{METHOD}

This is a methodological piece of research. It can be defined as research related to the investigation of methods related to acquiring, organizing, and analyzing data, and to the elaboration of an instrument to collect and analyze data related to the control of ISS. It will be performed in three stages: 
$\left(1^{\text {st }}\right)$ Integrative review of the literature, performed between March and April 2014, with over the time period 2004 to 2014. The databanks used were Lilacs and Mediline, accessed through the Health Virtual Library (BVS, in Portuguese) via PubMed, using descriptors in Portuguese (Procedimentos cirúrgicos operatórios; Infecção da Ferida Operatória; Fatores de Risco; Segurança do Paciente; Lista de Checagem) and the English translation of these same descriptors based on the Medical Subject Headings (MeSH): Surgical Wound Infection; Risk Factors; Patient Safety; Checklist.

$\left(2^{\text {nd }}\right)$ Development of the instrument, based on the evidence in the literature.

$\left(3^{\text {rd }}\right)$ Validation of the instrument at the researcher's workplace. The instrument developed will be used by specialized professionals at the institution, and tested on locations where pre-surgery, the surgery itself, and the post-operative care took place. As part of this stage, there will be a consensus conference, when the group will evaluate the content of the instrument, analyzing if the items selected are broad enou$\mathrm{gh}$, and if they are easily applicable.

The criteria for inclusion of professionals is based on having had three years' experience in the area of $\mathrm{CCH}$, possession of undergraduate diploma in nursing or medicine, and working in the institution where the instrument will be tested. Those workers who are absent or on vacation during the period of research, or those that did not participate in all the stages needed to validate the instrument are excluded. The group will consist of at least 10 professionals, bearing in mind the restrictions that the criteria for inclusion propose. It is forecast that this stage will be performed between January and March 2015. The research is approved by the Committee of Ethics in Research of the Medicine School of Fluminense Federal University, under protocol CAAE 31289314.0.0000.5243, approval protocol \#725.095.

\section{REFERENCES:}

1. World Health Organization (Switzerland). WHO. World Alliance for Patient Safety. Forward Programme 2008 - 2009.Geneva: WHO; 2008.

2. Ministério da Saúde (Brasil). Agência Nacional de Vigilância Sanitária. Sítio Cirúrgico. Critérios Nacionais de Infecções relacionadas à assistência à saúde. Brasília: Ministério da Saúde; 2009.

3. Silva LMG, Pawluk LC, Gebrim LH, Facina G, de-Gutiérrez MGR. Structure and process of nursing care for prevention of surgical site infection: observational study. Online braz j nurs [Internet]. 2009 January [Cited 2014 Sept 18]; 8 (1): . Available rom: http://www.objnursing.uff.br/index. php/nursing/article/view/1873.

All authors participated in the phases of this publication in one or more of the following steps, in According to the recommendations of the International Committee of Medical Journal Editors (ICMJE, 2013): (a) substantial involvement in the planning or preparation of the manuscript or in the collection, analysis or interpretation of data; (b) preparation of the manuscript or conducting critical revision of intellectual content; (c) approval of the versión submitted of this manuscript. All authors declare for the appropriate purposes that the responsibilities related to all aspects of the manuscript submitted to OBJN are yours. They ensure that issues related to the accuracy or integrity of any part of the article were properly investigated and resolved. Therefore, they exempt the OBJN of any participation whatsoever in any imbroglios concerning the content under consideration. All authors declare that they have no conflict of interest of financial or personal nature concerning this manuscript which may influence the writing and/or interpretation of the findings. This statement has been digitally signed by all authors as recommended by the ICMJE, whose model is available in http://www. objnursing.uff.br/normas/DUDE_eng_13-06-2013.pdf

Received: 08/29/2014

Revised: 09/19/2014

Approved: 09/19/2014 